\title{
Successful Treatment of Invasive Zygomycosis Based on a Prompt Diagnosis Using Molecular Methods in a Patient with Acute Myelogenous Leukemia
}

\author{
Junichiro Yuda ${ }^{1}$, Koji Kato ${ }^{1}$, Yoshikane Kikushige ${ }^{1}$, Kiyofumi Ohkusu ${ }^{2}$, Makiko Kiyosuke ${ }^{3}$, \\ Keiji Sakamoto ${ }^{1}$, Seido Oku ${ }^{1}$, Noriko Miyake ${ }^{1}$, Masako Kadowaki ${ }^{1}$, Tadafumi Iino ${ }^{4}$, \\ Kazuki Tanimoto ${ }^{1}$, Katsuto Takenaka ${ }^{1}$, Hiromi Iwasaki ${ }^{4}$, Toshihiro Miyamoto ${ }^{1}$, \\ Nobuyuki Shimono ${ }^{1}$, Takanori Teshima ${ }^{4}$ and Koichi Akashi ${ }^{1}$
}

\begin{abstract}
Zygomycosis is a lethal and invasive mold infection that is often associated with hematological malignancies. The keys for successful treatment include making a rapid diagnosis and appropriately administering antifungal agents. We herein report the early diagnosis of a case of zygomycosis in a patient with acute myeloid leukemia using a deoxyribonucleic acid sequence analysis. We successfully performed allogeneic hematopoietic stem cell transplantation with the use of high-dose liposomal amphotericin B and granulocyte transfusion.
\end{abstract}

Key words: zygomycosis, liposomal amphotericin B, stem cell transplantation, polymerase chain reaction

(Intern Med 53: 1087-1091, 2014)

(DOI: 10.2169/internalmedicine.53.1366)

\section{Introduction}

Zygomycosis is an invasive mold infection caused by members of the order Mucorales that frequently occurs in patients with hematological malignancies (1). Zygomycosis can present as rhinocerebral, pulmonary or disseminated disease with a rapid clinical course and lethal outcome (2). In patients with neutropenia, mortality due to zygomycosis increases significantly if antimicrobial treatment is either inappropriate or delayed. Therefore, the key aspects to treat zygomycosis include making a rapid diagnosis and appropriately administering antifungal agents. However, making a diagnosis based on histology remains challenging, as zygomycosis is rare and the causative agent has a morphology resembling that of yeast-like or filamentous fungi. We herein report a case of disseminated Rhizomucor pusillus infection in a patient with acute myeloid leukemia (AML). Although it is difficult to diagnose zygomycosis based on the results of a histopathological examination only, conducting a deoxyribonucleic acid (DNA) sequence analysis can lead to a rapid diagnosis and appropriate treatment of $R$. pusillus.

\section{Case Report}

A 49-year-old man was referred to our hospital with general fatigue and a history of splenectomy for idiopathic thrombocytopenia. The patient's white blood cell count was $3.3 \times 10^{9} / \mathrm{L}$, with $3 \%$ neutrophils and $6 \%$ myeloblasts, and he exhibited anemia ( $\mathrm{Hb}, 6.9 \mathrm{~g} / \mathrm{dL})$ and thrombocytopenia (platelet count, $2.2 \times 10^{9} / \mathrm{L}$ ) (Table). Bone marrow aspiration revealed an increase in myeloblasts (up to 20\%) with multilineage dysplasia, including a pseudo-Pelger-Huet anomaly in the myeloid lineage, megaloblastic changes in the erythroid lineage and the presence of micromegakaryocytes. The patient was diagnosed with AML with myelodysplasia-

\footnotetext{
${ }^{1}$ Department of Medicine and Biosystemic Science, Kyushu University Graduate School of Medical Science, Japan, ${ }^{2}$ Department of Microbiology, Gifu University Graduate School of Medicine, Japan, ${ }^{3}$ Department of Clinical Chemistry and Laboratory Medicine, Kyushu University Hospital, Japan and ${ }^{4}$ Center for Cellular and Molecular Medicine, Kyushu University Graduate School of Medical Science, Japan Received for publication July 13, 2013; Accepted for publication December 12, 2013 Correspondence to Dr. Koji Kato, kojikato@intmed1.med.kyushu-u.ac.jp
} 
Table. Laboratory Findings on Admission

\begin{tabular}{lclc}
\hline RBC & $188 \times 10^{4} / \mu \mathrm{L}$ & TP & $7.5 \mathrm{~g} / \mathrm{dL}$ \\
Hb & $6.9 \mathrm{~g} / \mathrm{dL}$ & Alb & $4.3 \mathrm{~g} / \mathrm{dL}$ \\
PIt & $2.2 \times 10^{4} / \mu \mathrm{L}$ & T-Bil & $0.3 \mathrm{mg} / \mathrm{dL}$ \\
WBC & $3230 / \mu \mathrm{L}$ & AST & $11 \mathrm{U} / \mathrm{L}$ \\
$\quad$ Neut & $4.0 \%$ & ALT & $10 \mathrm{U} / \mathrm{L}$ \\
Lymph & $85.5 \%$ & LDH & $259 \mathrm{U} / \mathrm{L}$ \\
$\quad$ Baso & $1.0 \%$ & ALP & $231 \mathrm{U} / \mathrm{L}$ \\
$\quad$ Eosino & $2.5 \%$ & BUN & $17.0 \mathrm{mg} / \mathrm{dL}$ \\
$\quad$ N-myelo & $1.0 \%$ & Cr & $0.76 \mathrm{mg} / \mathrm{dL}$ \\
Blast & $6.0 \%$ & Na & $141 \mathrm{mEq} / \mathrm{L}$ \\
& & $\mathrm{K}$ & $3.7 \mathrm{mEq} / \mathrm{L}$ \\
PT-TIME & $12.8 \mathrm{sec}$ & $\mathrm{Cl}$ & $101 \mathrm{mEq} / \mathrm{L}$ \\
PT-INR & $1.10 \mathrm{INR}$ & $\mathrm{CRP}$ & $1.08 \mathrm{~g} / \mathrm{dL}$ \\
APTT & $31.8 \mathrm{sec}$ & & \\
Fib & $337 \mathrm{mg} / \mathrm{dL}$ & & \\
FDP & $<2.6 \mu \mathrm{m} / \mathrm{mL}$ & & \\
D-dimer & $0.7 \mu \mathrm{g} / \mathrm{mL}$ & & \\
\hline
\end{tabular}

related changes (MRC) based on the World Health Organization 2008 classification. A cytogenetic analysis showed a complex karyotype, including monosomy 7 (Fig. 1). The administration of combined chemotherapy with idarubicin and cytarabine was immediately started as induction therapy against AML. After the completion of the chemotherapy regimen, the patient's neutropenia and fever continued despite the use of prophylactic antifungal treatment with fluconazole and broad-spectrum antibiotics. Cultures of blood, sputum, urine and stool were negative for fungi and bacteria, and the serum levels of galactomannan antigens and $\beta$ D-glucan were not elevated. However, on day 18 after the initiation of chemotherapy, the chest computed tomography (CT) findings showed a small nodular lesion in the upper right lobe of the lung. Treatment with intravenous voriconazole was initiated under a diagnosis of possible pulmonary invasive aspergillosis infection based on the European Organization for Research and Treatment of Cancer/Invasive Fungal Infections Cooperative Group and National Institute of Allergy and Infectious Diseases Mycoses Study Group criteria (3). Despite switching the medication regimen to voriconazole, the patient's general condition deteriorated in association with the development of hypoxia (oxygen saturation: $90-92 \%$ on room air). On day 28 after the initiation of chemotherapy, the CT findings demonstrated progressive pulmonary lesions with pleural effusion in the bilateral lungs, in addition to lesions in the kidney, liver, skin and brain (Fig. 2). Although a direct examination of cultured pleural effusion fluid using lactophenol cotton blue staining showed fungus without septate hyphae, classifying the genus and species of the organism was difficult microscopically (Fig. 3). Simultaneously, a molecular analysis of organisms in the pleural effusion was performed using polymerase chain reaction (PCR) targeting the internally transcribed spacer 1 (ITS1), 5.8S ribosomal DNA (rDNA) and ITS2 of the isolated fungus, according to a previously described method (4). Amplified PCR products of approximately 600 base pairs were sequenced and used to conduct a basic local alignment search of the GenBank sequence database (www. ncbi.nlm.nih.gov/blast/). The amplicon sequences were $100 \%$ identical to the corresponding sequences of the $R$. pusillus strain ATCC 46342 (GenBank accession number GU 256738). Based on the finding of invasive $R$. pusillus infection, treatment with intravenous liposomal amphotericin B (L-AmB) was initiated at a dose of $3 \mathrm{mg} / \mathrm{kg}$, which was promptly increased up to $10 \mathrm{mg} / \mathrm{kg}$, along with granulocyte colony-stimulating factor (G-CSF), which resulted in improvements in the patient's symptoms, including the recovery of neutrophils (Fig. 4). The patient achieved hematological complete remission (CR) after receiving the induction therapy. Because CT scans continued to show abnormal findings, such as abscesses in the brain, liver and kidney, in addition to pneumonia, the L-AmB therapy was continued to treat disseminated zygomycosis. The severity of these symptoms made it a priority to treat the disseminated zygomycosis over the AML with MRC (Fig. 5A).

On day 96 after the initiation of the induction therapy, the percentage of leukemic myeloblasts in the bone marrow had increased to $15 \%$. Despite the administration of low-dose cytarabine and aclacinon in order to decrease the leukemic burden, the patient failed to achieve $\mathrm{CR}$, and cytogenetic markers indicating a poor prognosis were detected, suggesting the need for immediate allogeneic hematopoietic stem cell transplantation (allo-HSCT) to achieve a cure. Certainly, the risk of exacerbating the disseminated zygomycosis was extremely high. However, we performed allo-HSCT in addition to the administration of high-dose L-AmB (10 mg/kg). The reduced-intensity conditioning regimen consisted of fludarabine (Flu; $\left.125 \mathrm{mg} / \mathrm{m}^{2}\right)$ and intravenous busulfan $(\mathrm{Bu}$; $12.8 \mathrm{mg} / \mathrm{kg}$ ). Graft-versus-host disease prophylaxis consisted of cyclosporin A and mycophenolate mofetil. The CD34 peripheral blood stem cells transplanted from a human leukocyte antigen-fully matched related donor (the patient's elderly brother) were administered at a dose of $6.6 \times 10^{6} / \mathrm{kg}$. In addition, G-CSF-mobilized granulocytes were deliberately transfused from the same allo-HSCT donor on days 2 and 3 and from the patient's son on days 8 and 9 after allo-HSCT. Engraftment of neutrophils was observed on day 12 after allo-HSCT (Fig. 4), and the disseminated zygomycosis was not exacerbated during the neutropenic period of allo-HSCT (Fig. 5B).

\section{Discussion}

Zygomycosis is a lethal opportunistic fungal complication observed in immunocompromised patients, particularly those with hematological malignancies, for which therapeutic options are limited. In patients with only localized infection, performing surgical excision and debridement is fundamental for a chance of cure. However, over the past decade, several new antifungal drugs suitable for clinical use and novel strategies for treating invasive fungal infection have been developed (5). Posaconazole and L-AmB are currently available and exhibit good activity against zygomycosis $(2,6)$, although voriconazole is not effective, as shown in the pre- 
(A)

(B)

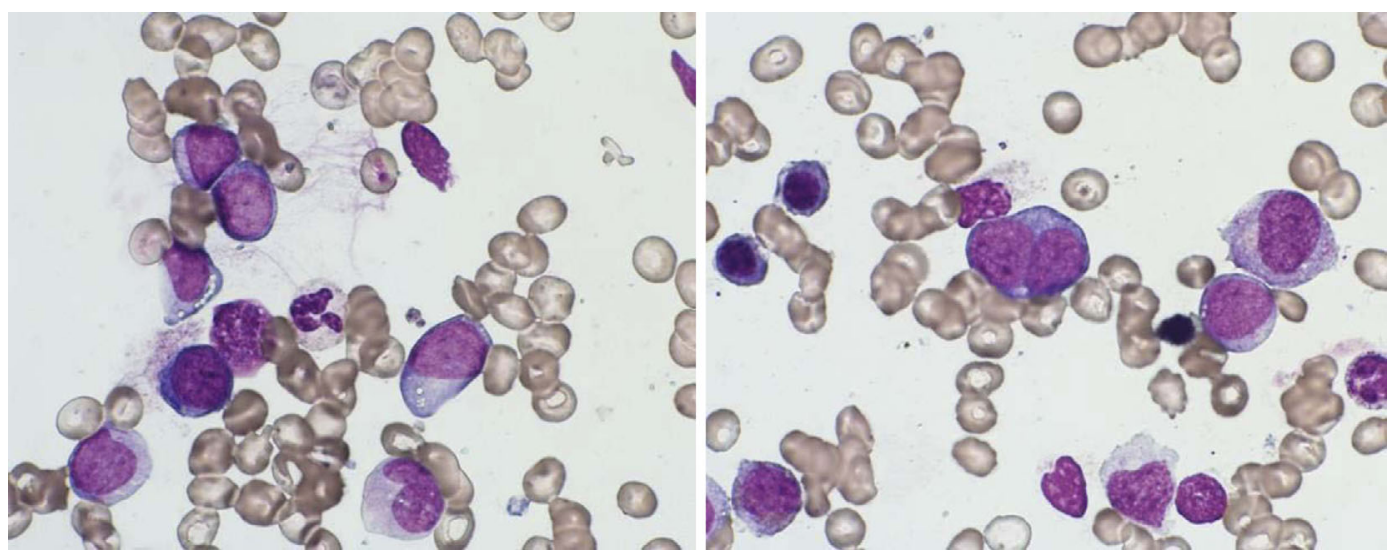

Figure 1. Bone marrow aspirates showing hypocellular marrow with myeloblasts (A) and multilineage dysplasia (B) (Wright-Giemsa stain, $\times 1,000)$. Giemsa-banded karyogram of the bone marrow cells, as follows: $45, \mathrm{XY}, \operatorname{dic}(3 ; 18)(\mathrm{p} 13 ; \mathrm{p11.2}), \operatorname{der}(5 ; 17)(\mathrm{p} 10 ; q 10),-7, \operatorname{dic}(13 ; 15)(\mathrm{p11} .2 ; \mathrm{P11.2}), \operatorname{add}(19)$ (p11), +21, idic(22)(p11.2).

(A)

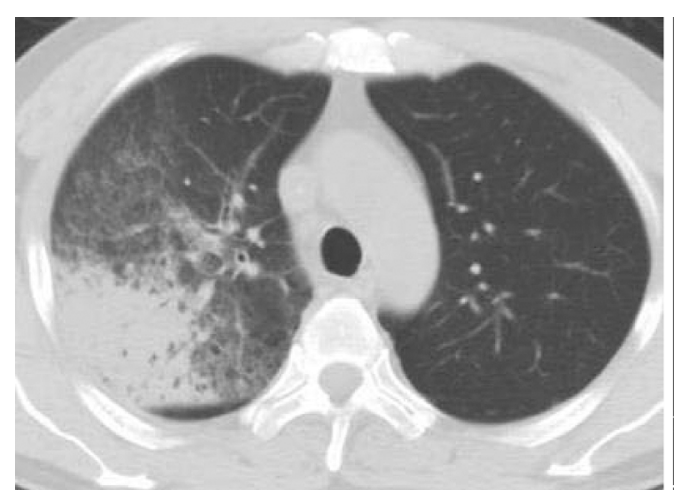

(B)

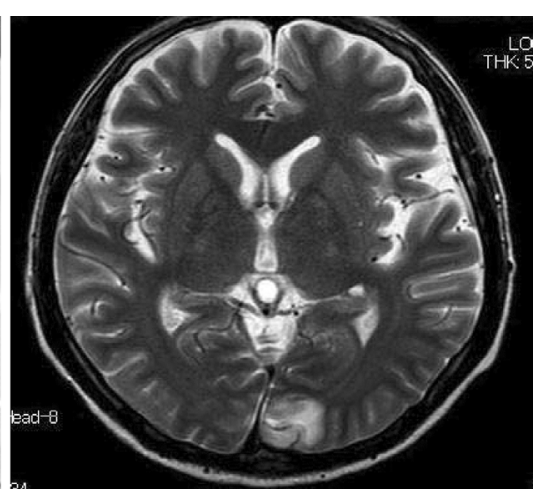

Figure 2. (A) High-resolution chest computed tomography revealed ground-glass opacity around a consolidated area in the right lobe. (B) T2-weighted magnetic resonance imaging demonstrated cerebral infarction in the left occipital lobe.

(A)

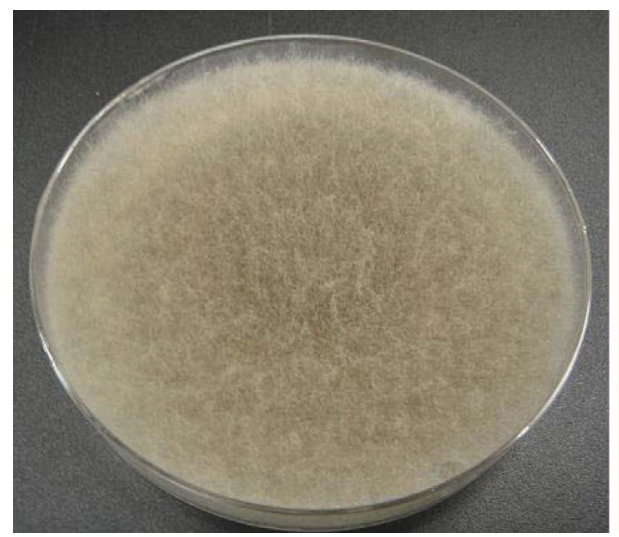

(B)

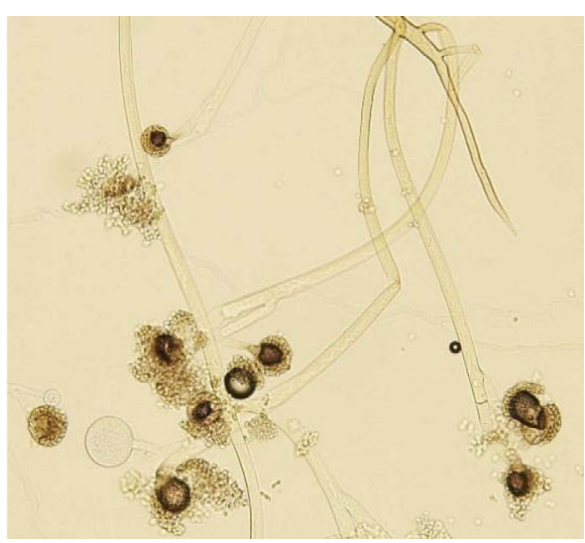

Figure 3. (A) Colonies formed from the pleural effusion fluid cultured on Sabouraud agar at $37^{\circ} \mathrm{C}$ for three days. (B) Photomicrograph of lactophenol cotton blue-stained fungi. 


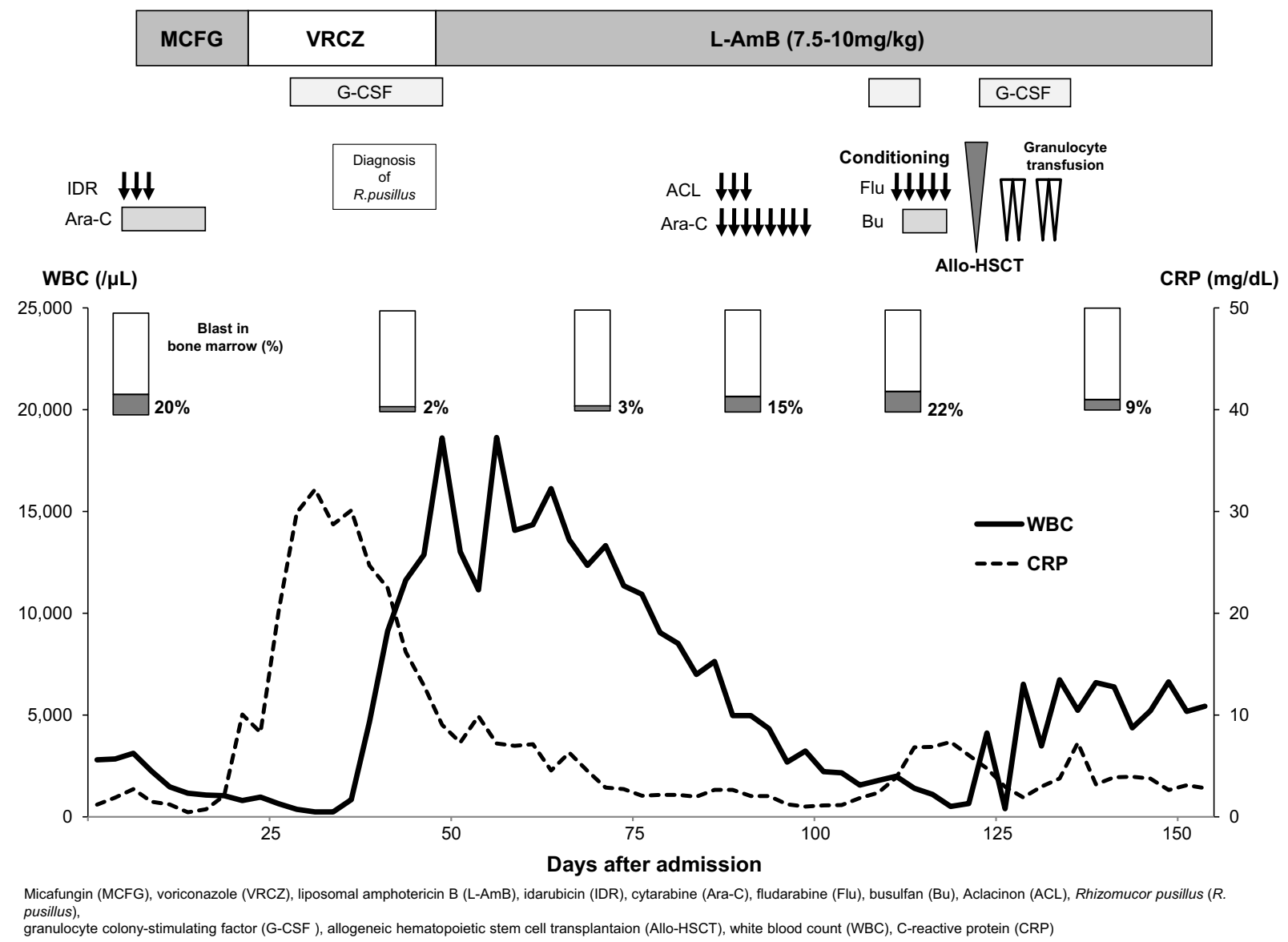

Figure 4. Clinical course of the anti-fungal therapy for zygomycosis during chemotherapy and allogeneic hematopoietic stem cell transplantation.

(A)

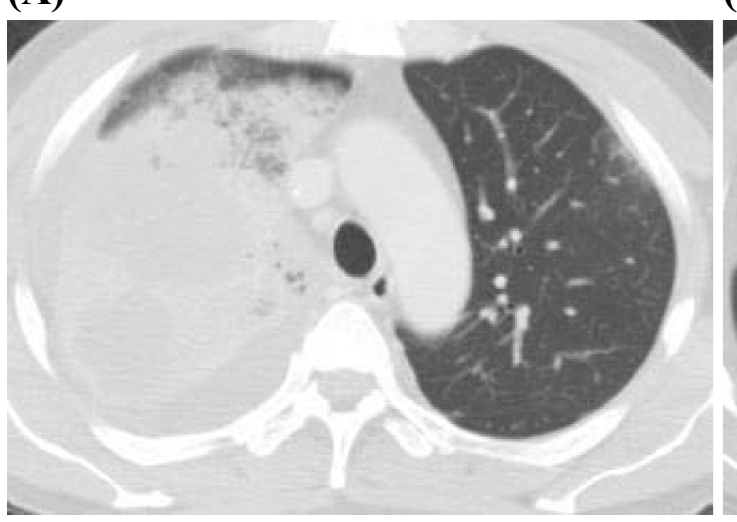

(B)

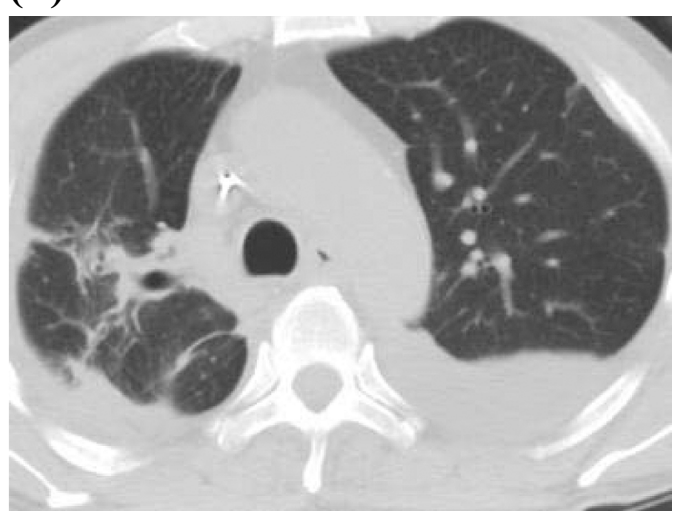

Figure 5. (A) High-resolution chest computed tomography (HRCT) disclosed consolidation and pleural effusion on day 63 after the initiation of induction therapy. (B) HRCT performed on day 37 after allo-HSCT showed an improvement of the lesion in the right lobe.

sent case. Because posaconazole has not yet been approved for use in Japan, L-AmB was the only choice of treatment in this case. L-AmB is available with less nephrotoxicity than conventional AmB; therefore, L-AmB can be administered safely at much higher doses $(10 \mathrm{mg} / \mathrm{kg})$, even in the setting of allo-HSCT. In addition, the transfusion of granulocytes likely decreases the risk of exacerbated invasive zygo- mycosis during the period of neutropenia that occurs after allo-HSCT $(7,8)$. Nevertheless, unlike invasive aspergillosis, zygomycosis is extremely rare, and the outcome of invasive zygomycosis treatment remains disappointing due to its association with a high mortality rate in immunocompromised patients (9).

In the present case, combined treatment consisting of the 
transfusion of high-dose L-AmB (2) and granulocytes (8) may account for the positive treatment outcome. However, the primary key to the successful treatment of disseminated zygomycosis was the rapid diagnosis of $R$. pusillus using the PCR method (10), which enabled the patient to undergo allo-HSCT in association with directed treatment comprising high-dose L-AmB, improving the prognosis and chance of survival (11). In general, diagnosing zygomycosis is more difficult than other fungal infections, as there are no specific clinical signs or biomarkers for identifying this fungal species (5). The serum levels of Aspergillus GM antigens or $\beta$ D-glucan are usually not elevated in patients with invasive zygomycosis. Therefore, conducting an early examination upon the suspicion of zygomycosis is very important, and tissue biopsies, the gold standard for diagnosis, should be performed in order to document zygomycosis when possible. Nevertheless, the rate of positive fungal cultures is usually low (12), and it is difficult to morphologically classify fungi to the species level. In this case, $R$. pusillus was detected in the pleural effusion, which is usually sterile, using PCR. We believe that PCR-based methods are useful for promptly confirming the classification of zygomycosis at the species level (13). In order to appropriately select antifungal agents, differentiating zygomycosis from more common opportunistic molds, such as Aspergillus, is also very important. Voriconazole, which is used as a first-line treatment for invasive pulmonary aspergillosis, does not exhibit good activity against zygomycosis (3). Cunninghamella species, which can cause zygomycosis with an aggressive clinical course, must be treated with high-dose L-AmB (14). Because molecular techniques are not widely clinically validated, they should be used to make a definitive diagnosis with caution in combination with morphological methods.

In conclusion, the correct identification and early diagnosis of invasive zygomycosis is necessary to initiate timely and appropriate antifungal therapy, as invasive zygomycosis can have a fatal outcome. The PCR method is useful for obtaining the appropriate diagnosis; however, further validation is required before this method can be routinely used in the clinical setting.

The authors state that they have no Conflict of Interest (COI).

\section{References}

1. Neofytos D, Horn D, Anaissie E, et al. Epidemiology and outcome of invasive fungal infection in adult hematopoietic stem cell transplant recipients: analysis of Multicenter Prospective Antifungal Therapy (PATH) Alliance registry. Clin Infect Dis 48: 265273, 2009.

2. Pagano L, Valentini CG, Caira M, Fianchi L. ZYGOMYCOSIS: current approaches to management of patients with haematological malignancies. Br J Haematol 146: 597-606, 2009.

3. Walsh TJ, Anaissie EJ, Denning DW, et al. Treatment of aspergillosis: clinical practice guidelines of the Infectious Diseases Society of America. Clin Infect Dis 46: 327-360, 2008.

4. Ferrer C, Colom F, Frases S, Mulet E, Abad JL, Alio JL. Detection and identification of fungal pathogens by PCR and by ITS2 and 5.8S ribosomal DNA typing in ocular infections. J Clin Microbiol 39: 2873-2879, 2001.

5. Kontoyiannis DP, Lewis RE. How I treat mucormycosis. Blood 118: 1216-1224, 2011.

6. Greenberg RN, Mullane K, van Burik JA, et al. Posaconazole as salvage therapy for zygomycosis. Antimicrob Agents Chemother 50: 126-133, 2006.

7. Pagano L, Valentini CG, Fianchi L, Caira M. The role of neutrophils in the development and outcome of zygomycosis in haematological patients. Clin Microbiol Infect 15 (Suppl 5): 33-36, 2009.

8. Grigull L, Beilken A, Schmid H, et al. Secondary prophylaxis of invasive fungal infections with combination antifungal therapy and G-CSF-mobilized granulocyte transfusions in three children with hematological malignancies. Support Care Cancer 14: 783-786, 2006.

9. Gonzalez CE, Rinaldi MG, Sugar AM. Zygomycosis. Infect Dis Clin North Am 16: 895-914, vi, 2002.

10. Kasai M, Harrington SM, Francesconi A, et al. Detection of a molecular biomarker for zygomycetes by quantitative PCR assays of plasma, bronchoalveolar lavage, and lung tissue in a rabbit model of experimental pulmonary zygomycosis. J Clin Microbiol 46: 3690-3702, 2008.

11. Aoki $\mathrm{T}$, Kamezaki $\mathrm{K}$, Miyamoto $\mathrm{T}$, et al. Cord blood stem cell transplantation in a patient with disseminated mucormycosis and acute myelogenous leukemia. Transpl Infect Dis 12: 277-279, 2010.

12. Tarrand JJ, Han XY, Kontoyiannis DP, May GS. Aspergillus hyphae in infected tissue: evidence of physiologic adaptation and effect on culture recovery. J Clin Microbiol 43: 382-386, 2005.

13. Rickerts V, Mousset S, Lambrecht E, et al. Comparison of histopathological analysis, culture, and polymerase chain reaction assays to detect invasive mold infections from biopsy specimens. Clin Infect Dis 44: 1078-1083, 2007.

14. Kimura M, Araoka $\mathrm{H}$, Uchida $\mathrm{N}$, et al. Cunninghamella bertholletiae pneumonia showing a reversed halo sign on chest computed tomography scan following cord blood transplantation. Med Mycol 50: 412-416, 2012.

(C) 2014 The Japanese Society of Internal Medicine

http://www.naika.or.jp/imonline/index.html 